\title{
On Evaluating Parallel Sparse Cholesky Factorizations
}

\author{
Wen-Yang Lin and Chuen-Liang Chen \\ Department of Computer Science and Information Engineering \\ National Taiwan University \\ Taipei, TAIWAN \\ e-mail: clchen@csie.ntu.edu.tw
}

\begin{abstract}
Though many parallel implementations of sparse Cholesky factorization with the experimental results accompanied have been proposed, it seems hard to evaluate the performance of these factorization methods theoretically because of the irregular structure of sparse matrices. This paper is an attempt to such research. On the basis of the criteria of parallel computation and communication time, we successfully evaluate four widely adopted Cholesky factorization methods, including column-Cholesky, row-Cholesky, submatrix-Cholesky and multifrontal. The results show that the multifrontal method is superior to the others.
\end{abstract}

\section{Introduction}

In the directive solution of a sparse symmetric and positive definite linear system $A x=b$, Cholesky factorization has acted as a synonym of the decomposition of $A$ into $L L^{T}$, for $L$ a lower triangular. As the timeconsuming characteristic of Cholesky factorization and the advance of parallel computer architectures, a demand for efficient parallel sparse Cholesky factorization algorithms have emerged.

Though a variety of parallel sparse Cholesky factorizations have been proposed [7] and usually accompanied with some experimental results, it still lacks of theoretical evaluation due to the irregularity of sparse matrices. The evaluation not only serves as a prediction of the actual performance, but also exploits the potentiality and limitation of a Cholesky factorization; thus helps in choosing the appropriate method for a particular machine. This paper is an attempt to such study. On the basis of the criteria of parallel computation and communication time, we consider four widely adopted Cholesky factorization methods, including column-Cholesky, rowCholesky, submatrix-Cholesky and multifrontal, on distributed-memory multiprocessors. With some graphtheoretic techniques we have succeed in the evaluation and found that multifrontal method is superior to the others.

The remainder of this paper is organized as follows. In Section 2, we review some graph-theoretic notions used in matrix computation and the four Cholesky factorizations. In Section 3, we define the parallel computation and communication criteria, and formulate them in terms of graph notations. On the basis of the criteria, we evaluate the performance of these four Cholesky factorizations in Section 4. Experiments on some practical sparse matrices are also included. Section 5 gives the conclusions.

\section{Background}

\subsection{Graph model for Cholesky factorizations}

In this subsection, we briefly review the combinatorial aspect of the elimination process as formulated by Rose [12] and then confine it to a more specific graph model on which later derivations will be based.

Let $A=\left(a_{i j}\right)$ be an $n \times n$ sparse symmetric positive definite matrix. The associated graph of $A, G_{A}=\left(V_{A}, E_{A}\right)$, is constructed as below:

$$
\begin{aligned}
& V_{A}=\left\{v_{i} \mid v_{i} \text { corresponds to column/row } i \text { of } A, 1 \leq i \leq n\right\}, \\
& E_{A}=\left\{\left(v_{i}, v_{j}\right) \mid a_{i j} \neq 0,1 \leq i<j \leq n\right\} .
\end{aligned}
$$

The symmetric Gaussian elimination of $A$ can be described as follows. Letting

$$
A=A^{(0)}=\left[\begin{array}{ll}
d & r^{T} \\
r & \bar{H}
\end{array}\right]
$$

where $d$ is a positive scalar, $r$ is an $(n-1) \times 1$ and $\bar{H}$ is an $(n-1) \times(n-1)$ matrix, the first step of symmetric elimination is the factorization

$$
A^{(0)}=\left[\begin{array}{cc}
\sqrt{d} & 0 \\
\frac{r}{\sqrt{d}} & I_{n-1}
\end{array}\right]\left[\begin{array}{cc}
1 & 0 \\
0 & \widetilde{H}-\frac{r r^{T}}{d}
\end{array}\right]\left[\begin{array}{cc}
\sqrt{d} & \frac{r^{T}}{\sqrt{d}} \\
0 & I_{n-1}
\end{array}\right] .
$$

Letting $A^{(1)}=\bar{H}-r r^{T} / d$, the factorization is then completed by recursively applying the basic step in (1) to $A^{(1)}$, $A^{(2)}$, and so on. Since $A$ is sparse, some initially zero 
entries in $A$ may become nonzero in $L$ during factoring $A$ into $L L^{T}$, which are called fills or fill-ins.

As observed by Rose, symmetric Gaussian elimination can be interpreted by a sequence of elimination graphs

$$
G_{A}=G_{A^{(0)}} \rightarrow G_{A^{(n)}} \rightarrow \cdots \rightarrow G_{A^{(n-1)}} \rightarrow G_{A^{(n)}}=\varnothing .
$$

where graph $G_{A^{(i)}}$ of $A^{(i)}$ is obtained from that of $A^{(i-1)}$ by (i) deleting $v_{i}$ and its incident edges from $G_{A^{(-1)} \text {, and (ii) }}$ adding edges to $G_{A^{(j-1)}}$ so that nodes adjacent to $v_{i}$ are pairwise adjacent in $G_{A}^{(\text {) }}$.

For our purpose we confine the above graph model to a more specific one. Let $G_{F}$ be the filled graph of $G_{A}$, where $F=L+L^{T}$ denotes the filled matrix of $A$, i.e.,

$$
G_{F}=\bigcup_{i=0}^{n} G_{A^{(i)}} \text {. }
$$

It is meaningful since the filled matrix of $A$ has to be determined before the numerical factorization proceeds. So, we refer the factoring process of $A$ as a sequence of elimination graphs of $G_{F}$ such that from $G_{F^{(j-1)}}$ to $G_{F^{(m)}}$ only $v_{i}$ and its incident edges are eliminated since all nodes adjacent to $v_{i}$ has been pairwise connected.

\subsection{Parallel sparse Cholesky factorizations}

It is well-known that the algorithmic form of Cholesky factorization of $A$ can be viewed as a triple nested loop containing the following statement [1]

$$
a_{i j} \leftarrow a_{i j}-l_{i k} l_{j k}
$$

where $L=\left(l_{i j}\right)$ is the Cholesky factor.

Depending on which of the three indices is placed at the outmost loop there are three basic forms [4]: columnCholesky, row-Cholesky, and submatrix-Cholesky. In some articles the column-Cholesky and submatrixCholesky are also known as fan-in and fan-out respectively. Recently, a sophisticated variant of the submatrixCholesky factorization, called multifrontal method [2], has been proposed and soon became a competitor to the other Cholesky factorizations. This paper is devoted to these four algorithms.

To exploit the potential parallelism existing in sparse matrix factorization, a commonly used structure is elimination tree [13]. Duff [3] has shown that the elimination tree also can be acted as an assembly tree for multifrontal method. An elimination tree $T_{A}$ associated with the Cholesky factor $L$ of matrix $A$ is a tree containing the same nodes as the filled graph of $A$ and for each $v_{k}$ with $k$ $<n$, its parent node is $v_{p}=\operatorname{parent}\left(v_{k}\right)$, where $p=\min |j|$ $j>k$ and $\left.l_{j k} \neq 0\right\}$. A related definition of parent is child, where $\operatorname{child}\left(v_{k}\right)=\left\{v_{c} \mid\right.$ parent $\left.\left(v_{c}\right)=v_{k}\right\}$. On the basis of the elimination tree model, we describe in Algorithms 1 to 4 respectively the parallel sparse column-, row-, submatrix-Cholesky and multifrontal methods, which are considered on a distributed-memory multiprocessor with the following assumptions:

(1) There is an unlimited number of processors that connected via a network of sufficiently wide bandwidth.

Algorithm 1. Column-Cholesky factorization

for $j:=1$ to $n$ do in parallel

while $v_{j}$ is not a leaf node do waiting;

for $k:=1$ to $j-1$ and $l_{j k} \neq 0$ do

for $i:=j$ to $n$ and $l_{i k} \neq 0$ do

$l_{i j} \leftarrow \sqrt{a_{i j}} ;$

for $i:=j+1$ to $n$ and $a_{i j} \neq 0$ do

$$
l_{i j} \leftarrow a_{i j} / l_{i j}
$$

eliminate node $v_{j}$ from the elimination tree;

Algorithm 2. Row-Cholesky factorization.

for $i:=1$ to $n$ do in parallel

while $v_{i}$ is not a leaf node do waiting;

for $k:=1$ to $i-1$ and $a_{i k} \neq 0$ do

$$
l_{i k} \leftarrow a_{i k} / l_{k k}
$$$$
\text { for } j:=k+1 \text { to } i \text { and } l_{j k} \neq 0 \text { do }
$$

$l_{i i} \leftarrow \sqrt{a_{i i}} a_{i j} \leftarrow a_{i j}-l_{i k} l_{j k} ;$

eliminate node $v_{i}$ from the elimination tree;

Algorithm 3. Submatrix-Cholesky factorization.

for $k:=1$ to $n$ do in parallel

while $v_{k}$ is not a leaf node do waiting;

for $j:=k$ to $n$ and $a_{j k} \neq 0$ do

$$
a_{j k} \leftarrow a_{j k}+\sum_{i<k \text { and } l_{k i} \neq 0} t_{j k}^{(i)} ;
$$

$l_{k k} \leftarrow \sqrt{a_{k k}}$;

for $j:=k+1$ to $n$ and $a_{j k} \neq 0$ do

$$
l_{j k} \leftarrow a_{j k} / l_{k k}
$$

for $j:=k+1$ to $n$ and $l_{j k} \neq 0$ do

$$
\text { for } i:=k+1 \text { to } n \text { and } l_{i k} \neq 0 \text { do }
$$

$$
t_{i j}^{(k)} \leftarrow-l_{i k} l_{j k} \text {; }
$$

eliminate node $v_{k}$ from the elimination tree;

Algorithm 4. Multifrontal method.

for $k:=1$ to $n$ do in parallel

while $v_{k}$ is not a leaf node do waiting;

create frontal matrix $F^{(k)} \leftarrow A_{*_{k}}+\sum_{v_{c} \in \operatorname{child}\left(v_{k}\right)} \bar{F}^{(c)}$;

apply a sequential dense Cholesky to factor the first column $F_{* 1}^{(k)}$ of $F^{(k)}$;

$L_{* k} \leftarrow F_{* 1}^{(k)}$;

$\bar{F}^{(k)} \leftarrow F^{(k)}-F_{* 1}^{(k)}$;

eliminate node $v_{k}$ from the elimination tree; 
(2) The column-oriented distribution is used for column-, submatrix-Cholesky and multifrontal; roworiented distribution is used for row-Cholesky. Each processor is solely responsible for the task of maintaining and updating its column or row.

(3) For simplicity, we ignore the underlying interconnecting and routing topology, and assume a message (a column or a row) can be transferred within a fixed time.

In order to keep the Cholesky methods consistent in generic form, the communication is assumed to be invoked by the computation without an explicit indication. The multifrontal method, however, is rather complicated, and so we give only an informal description; details can be found in the original paper by Duff and Reid [2]. In Algorithm 3, notation $t_{i j}^{(k)}$ denotes an intermediate updated factor that is generated in the factorization of column $k$ and will be accumulated to entry $a_{i j}$ when factoring column $j$, for $j>k$. In Algorithm 4, $F^{(k)}$ represents the frontal matrix associated with column $k$ and $\bar{F}^{(k)}$ the remaining frontal matrix after the removal of the first column; $A_{*_{k}}$ and $L_{*_{k}}$ simply denote column $k$ of $A$ and $L$, respectively.

\section{The evaluation criteria}

\subsection{Definition}

In $[9,10]$ the authors have defined two evaluation criteria, parallel computation time and parallel communication time, to discuss the ordering problem, which indeed represent the computation and communication times needed to complete a Cholesky factorization in parallel. We adopt these two criteria and, for selfcontained, we repeat the definitions in the following.

For $v_{i}, 1 \leq i \leq n$, let $m t p\left(v_{i}\right)$ denote the number of multiplicative operations (square root, multiplication, and division) and $m s g\left(v_{i}\right)$ the number of messages (columns or rows) acquired by $v_{i}$. The parallel computation time and communication time to complete node $v_{i}$ are corresponding to the cost of a critical path from some leaf node to $v_{i}$, i.e.,

$$
\begin{aligned}
\operatorname{Comp}\left(v_{i}\right) & = \begin{cases}m t p\left(v_{i}\right), & \text { if } v_{i} \text { is leaf } \\
m t p\left(v_{i}\right)+\text { Mchild_Comp }\left(v_{i}\right), & \text { otherwise }\end{cases} \\
\operatorname{Comm}\left(v_{i}\right) & = \begin{cases}m s g\left(v_{i}\right), & \text { if } v_{i} \text { is leaf } \\
m s g\left(v_{i}\right)+\text { Mchild_Comm }\left(v_{i}\right), & \text { otherwise }\end{cases}
\end{aligned}
$$

where

Mchild_Comp $\left(v_{i}\right)=\max \left\{\operatorname{Comp}(x) \mid x \in \operatorname{child}\left(v_{j}\right)\right\}$,

$\operatorname{Mchild} \_\operatorname{Comm}\left(v_{i}\right)=\max \left\{\operatorname{Comm}(x) \mid x \in \operatorname{child}\left(v_{i}\right)\right\}$.

Then the parallel computation time and communication time required to complete the factorization, denoted as Comp and Comm, are equal to $\operatorname{Comp}\left(v_{n}\right)$ and $\operatorname{Comm}\left(v_{n}\right)$ respectively. Moreover, we introduce abbreviations, $C$,
$R, S$ and $M$ as subscripts to distinguish different Cholesky factorizations, e.g., $m t p_{s}(v)$ represents the number of multiplicative operations associated with node $v$ under submatrix-Cholesky factorization.

\subsection{Formulation of the criteria}

To study the behavior of and to distinguish the superiority among various Cholesky factorizations, we have to formulate the criteria.

Consider the filled graph $G_{F}$ of matrix $A$. For each node $v$ in $G_{F}$, we denote its adjacent set as $\operatorname{adj}_{G_{F}}(v)$ and its degree as $\operatorname{deg}_{G_{y}}(v)$, where $\operatorname{deg}_{G_{z}}(v)=\left|a d j_{G_{F}}(v)\right|$. The prior (monotone) adjacent set $\operatorname{Padj}_{G_{f}}(v)\left(\operatorname{Madj}_{G_{r}}(v)\right)$ is the set of all nodes adjacent to and numbered lower (higher) than $v ; P \operatorname{Pdeg}_{G_{F}}(v)\left(M \operatorname{deg}_{G_{F}}(v)\right)$ denote the size of $\operatorname{Padj}_{G_{p}}(v)\left(\operatorname{Madj}_{G_{p}}(v)\right)$. In what follows all discussions are related to $G_{F}$ we thus omit the subscript, $G_{F}$ in the above notations for simplicity.

A clique is a set of nodes with the property that all its members are pairwise adjacent; moreover, if no other node can be added while preserving the pairwise adjacent property, then the clique is called maximal. Assume $G_{F}$ comprises $K_{1}, K_{2}, \ldots, K_{q}$ maximal cliques. A maximal clique $K_{j}, 1 \leq j \leq q$, might change as the elimination proceeds and so similar to $G_{F^{(t-1)}}$ we denote the residual clique [8] of $K_{j}$ after the $i$ th elimination step as $K_{j}^{(i)}$. More precisely,

$$
K_{j}^{(i)}= \begin{cases}K_{j}^{(i-1)}-\left\{v_{i}\right\}, & \text { if } v_{i} \in K_{j}^{(i-1)}, \\ K_{j}^{(i-1)}, & \text { otherwise. }\end{cases}
$$

It should be noticed that a residual clique is not necessarily maximal. Furthermore, for node $v_{i}$ (remember it is corresponding to column or row $i$ of $A$ and is eliminated in the $i$ th step) we define $\Omega\left(v_{i}\right)$ as the identity of the maximal residual clique that contains $v_{i}$ when $v_{i}$ is eliminated. In other words, $K_{\Omega\left(v_{i}\right)}^{(i-1)}$ is the only maximal resid-

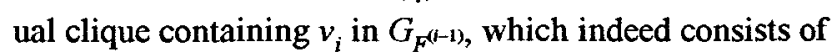
the set of nodes adjacent to and ordered after $v_{i}$ plus $v_{i}$, i.e., $K_{\Omega\left(v_{i}\right)}^{(i-1)}=\operatorname{Madj}\left(v_{i}\right) \cup\left\{v_{i}\right\}$ and thus $\left|K_{\Omega\left(v_{i}\right)}^{(i-1)}\right|=M \operatorname{deg}\left(v_{i}\right)+1$. The notion $K_{\Omega\left(v_{i}\right)}^{(j)}$, for $1 \leq j \leq n$, then represents the residual clique of $K_{\Omega\left(v_{i}\right)}$.

The following equalities are useful for our derivations.

$$
\begin{aligned}
& \operatorname{Padj}\left(v_{j}\right)=\left\{v_{k} \mid 1 \leq k \leq j-1 \text { and } l_{j k} \neq 0\right\} \\
& \sum_{j \leq i \leq n, l_{k} \neq 0}^{1}=\left|K_{\Omega\left(v_{k}\right)}^{(j-1)}\right| \text {, for } k \leq j-1 \\
& \left|K_{\Omega\left(v_{i}\right)}^{(i)}\right|=\left|K_{\Omega\left(v_{i}\right)}^{(i-1)}\right|-1
\end{aligned}
$$

Lemma 1. The numbers of multiplicative operations associated with node $v_{j}$ in $T_{A}$ for column-, row-, submatrix-Cholesky, and multifrontal factorizations can be individually formulated as 


$$
\begin{aligned}
& m t p_{C}\left(v_{i}\right)=\sum_{v_{j} \in P a d j\left(v_{i}\right) \cup\left\{v_{i}\right\}}\left|K_{\Omega\left(v_{j}\right)}^{(i-1)}\right|, \\
& m t p_{R}\left(v_{i}\right)=\sum_{v_{j} \in \operatorname{Padj}\left(v_{i}\right) \cup\left\{v_{j}\right\}}\left(\left|K_{\Omega\left(v_{j}\right)}^{(j-1)}\right|-\left|K_{\Omega\left(v_{j}\right)}^{(i)}\right|\right), \\
& m t p_{S}\left(v_{i}\right)=m t p_{M}\left(v_{i}\right)=\sum_{v_{j} \in \operatorname{Madj}\left(v_{i}\right) \cup\left\{v_{j}\right\}}\left|K_{\Omega\left(v_{j}\right)}^{\left(j-v^{\prime}\right)}\right| .
\end{aligned}
$$

Proof. We prove the case of $m t p_{C}\left(v_{i}\right)$ first. For each iteration of "for $j$ " loop in Algorithm 1, there are $\sum_{1 \leq k \leq j-1, l_{j k \neq} 0} \sum_{j \leq i \leq n, l_{k \neq} 0} 1$ multiplications, one square root, and $\sum_{j+1 \leq i \leq n, l_{k}, 0} 1$ divisions. Totally, the number of multiplicative operations is:

$$
\begin{aligned}
& \sum_{\substack{1 \leq k \leq j-1 \\
l_{j k} \neq 0}} \sum_{\substack{j \leq i \leq n \\
k \neq 0}} 1+1+\sum_{\substack{j+1 \leq i \leq n \\
l_{j} \neq 0}} 1 \\
= & \sum_{\substack{1 \leq k \leq i-1 \\
l_{j k} \neq 0}}\left|K_{\Omega\left(v_{k}\right)}^{(j-1)}\right|+1+\left|K_{\Omega\left(v_{j}\right)}^{(j)}\right|, \text { by (3) } \\
= & \sum_{v_{k} \in \operatorname{Padj}\left(v_{j}\right)}\left|K_{\Omega\left(v_{k}\right)}^{(j-1)}\right|+\left|K_{\Omega\left(v_{j}\right)}^{(j-1)}\right|, \text { by (2) and (4) } \\
= & \sum_{v_{k} \in \operatorname{Padj(v_{j})}\left(v_{j}\right\}}\left|K_{\Omega\left(v_{k}\right)}^{(j-1)}\right| .
\end{aligned}
$$

The lemma then follows by simple index substitution.

As for other cases, we can prove them by observing Algorithms 2, 3, 4, and similar derivations.

For submatrix-Cholesky and multifrontal factorizations, their $m t p$ 's are the same so we will denote that as $m t p_{S M}\left(v_{i}\right)$. Furthermore, be aware that $\operatorname{Madj}\left(v_{i}\right) \cup\left\{v_{i}\right\}=$ $K_{\Omega\left(v_{i}\right)}^{(i-1)}$ and thus

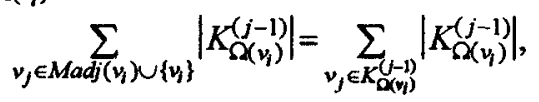

whose structure corresponds to a dense lower triangular matrix. Henceforth, we can rewrite the formula as

$$
\begin{aligned}
m t p_{S M}\left(v_{i}\right) & =\frac{1}{2}\left|K_{\Omega\left(v_{i}\right)}^{(i-1)}\right|\left(\left|K_{\Omega\left(v_{i}\right)}^{(i-1)}\right|+1\right) \\
& =\frac{1}{2}\left(\operatorname{Mdeg}\left(v_{i}\right)+1\right)\left(\operatorname{Mdeg}\left(v_{i}\right)+2\right) .
\end{aligned}
$$

Lemma 2. The numbers of messages (columns or rows) acquired to update node $v_{j}$ in $T_{A}$ for column-, row-, submatrix-Cholesky, and multifrontal factorizations can be individually formulated as

$$
\begin{aligned}
& m s g_{C}\left(v_{i}\right)=m s g_{R}\left(v_{i}\right)=m s g_{S}\left(v_{i}\right)=P \operatorname{deg}\left(v_{i}\right), \\
& m s g_{M}\left(v_{i}\right)=\sum_{v_{j} \in \operatorname{child}\left(v_{i}\right)} \operatorname{Mdeg}\left(v_{j}\right) .
\end{aligned}
$$

Proof. The derivation is similar to Lemma 1 by observing the remote accessing data in Algorithms 1 to 4 and applying the corresponding graph notations.

For column-, row-, and submatrix-Cholesky factorizations, their $m s g$ 's are the same so we will denote that as $m s g_{C R S}\left(v_{i}\right)$.

\section{Evaluation}

\subsection{Theoretical results}

In this subsection, we derive the evaluation of the four Cholesky factorization algorithms. A useful property quoted from Schreiber [13] is stated first.

Lemma 3. (Schreiber [13]) For each node $v_{j}, 1 \leq j<$ $n, \operatorname{Madj}\left(v_{j}\right)$ is a subset of nodes on the path from $v_{j}$ to the root $v_{n}$ of $T_{A}$.

Theorem 4. On solving a given sparse matrix, the computation time of submatrix-Cholesky (multifrontal) factorization is no more than that of column-Cholesky factorization.

Proof. This theorem can be proven if we can prove that for any path, say Path, from a leaf to the root on any elimination tree,

$$
\sum_{v_{j} \in \text { Path }} m t p_{C}\left(v_{j}\right) \geq \sum_{v_{i} \in P a t h} m t p_{S M}\left(v_{i}\right) \text {. }
$$

By Lemmas 1, 3 and the definitions of Madj and Padj, we have the following derivations:

$$
\begin{aligned}
& \sum_{v_{j} \in \text { Path }} m t p_{C}\left(v_{j}\right)=\sum_{v_{j} \in P a t h v_{j} \in P a d j\left(v_{j}\right) \cup\left\{v_{j}\right\}}\left|K_{\Omega\left(v_{i}\right)}^{(j-1)}\right| \\
& =\sum_{v_{j} \in P a t h} \sum_{\substack{v_{i} \in P a t h \\
\& v_{i} \in P a d j\left(v_{j}\right) \cup\left\{v_{j}\right\}}}\left|K_{\Omega\left(v_{j}\right)}^{(j-1)}\right|+\sum_{v_{j} \in P a t h} \sum_{\substack{v_{i} \notin P a t h \\
\& v_{i} \in P a d j\left(v_{j}\right)}}\left|K_{\Omega\left(v_{i}\right)}^{(j-1)}\right| \\
& \geq \sum_{v_{j} \in P a t h} \sum_{\substack{v_{j} \in P a t h \\
\& v_{i} \in P a d j\left(v_{j}\right) \cup\left\{v_{j}\right\}}}\left|K_{\Omega\left(v_{i}\right)}^{(j-1)}\right| \\
& =\sum_{v_{j} \in \text { Path }} \sum_{\substack{v_{j} \in P \text { ath } \\
\& v_{j} \in \operatorname{Madj}\left(v_{i}\right) \cup\left\{v_{i}\right\}}}\left|K_{\Omega\left(v_{i}\right)}^{(j-1)}\right| \\
& =\sum_{v_{i} \in P a t h v_{j} \in M a d j\left(v_{i}\right) \cup\left\{v_{l}\right\}}\left|K_{\Omega\left(v_{i}\right)}^{(j-1)}\right|=\sum_{v_{i} \in P a t h} m t p_{S M}\left(v_{i}\right)
\end{aligned}
$$

Theorem 5. On solving a given sparse matrix, the computation time of submatrix-Cholesky (multifrontal) factorization is no more than that of row-Cholesky factorization.

Proof. This theorem can be proven if we can prove that for any path, say Path, from a leaf to the root on any elimination tree,

$$
\sum_{v_{j} \in \text { Path }} m t p_{R}\left(v_{j}\right) \geq \sum_{v_{i} \in \text { Path }} m t p_{S M}\left(v_{i}\right)
$$

By Lemmas 1, 3 and the definitions of Madj and Padj, we have the following derivations:

$$
\begin{aligned}
& \sum_{v_{j} \in \text { Path }} m t p_{R}\left(v_{j}\right) \\
& =\sum_{v_{j} \in P a t h} \sum_{\substack{v_{i} \in P_{a t h} \\
\& v_{i} \in \operatorname{Padj}^{\prime}\left(v_{j}\right) \cup\left\{v_{j}\right\}}}\left(\left|K_{\Omega\left(v_{i}\right)}^{(i-1)}\right|-\left|K_{\Omega\left(v_{i}\right)}^{(j)}\right|\right)
\end{aligned}
$$

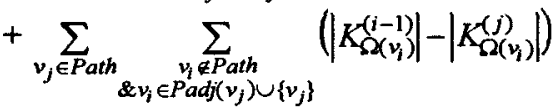

$$
\begin{aligned}
& \geq \sum_{v_{j} \in P a t h} \sum_{\substack{v_{i} \in P a t h \\
\& v_{j} \in P_{a d j\left(v_{j}\right) \cup\left(v_{j}\right\}}}}\left(\left|K_{\Omega\left(v_{i}\right)}^{(i-1)}\right|-\left|K_{\Omega\left(v_{i}\right)}^{(j)}\right|\right) \\
& =\sum_{v_{i} \in P a t h} \sum_{\substack{v_{j} \in P a t h \\
\& v_{j} \in \operatorname{Madj}\left(v_{i}\right) \cup\left\{v_{i}\right\}}}\left(\left|K_{\Omega\left(v_{i}\right)}^{(i-1)}\right|-\left|K_{\Omega\left(v_{i}\right)}^{(j)}\right|\right)
\end{aligned}
$$




$$
=\sum_{v_{i} \in P a t h} \sum_{\substack{v_{j} \in P_{\text {ath }} \\ \text { \& } v_{j} \in \operatorname{Madj}\left(v_{j}\right) \cup\left\{v_{i}\right\}}}\left|K_{\Omega\left(v_{i}\right)}^{(j-1)}\right|=\sum_{v_{i} \in \operatorname{Path}} m t p_{S M}\left(v_{i}\right)
$$

In derivations, note that, for each node $v_{i}$ on the Path, $\left.\sum_{v_{j} \in \text { Path\& } v_{j} \in \operatorname{Madj}\left(v_{i}\right) \cup\left\{v_{i}\right\}}\left(\left|K_{\Omega\left(v_{i}\right)}^{(i-1)}\right|-\mid K_{\Omega\left(v_{i}\right)}^{(j)}\right)\right)$ is counted in decreasing order and $\sum_{v_{j} \in \operatorname{Path}_{j} v_{j} \in \operatorname{Madj}\left(v_{1}\right) \cup\left\{v_{1}\right\}}\left|K_{\Omega\left(v_{1}\right)}^{(j-1)}\right|$ is counted in increasing order, however their summations are the same.

Theorem 6. On solving a given sparse matrix, the computation time of column-Cholesky factorization is no more than that of row-Cholesky factorization.

Proof. By observing derivations on the proofs of Theorems 4 and 5, this theorem will be true if we can prove that:

$$
\begin{aligned}
& \sum_{v_{j} \in P \text { Path }} \sum_{\substack{v_{i} \notin \operatorname{Path} \\
\& v_{i} \in \operatorname{Pad}\left(v_{j}\right)}}\left|K_{\Omega\left(v_{j}\right)}^{(j-1)}\right| \\
& \leq \sum_{v_{j} \in P a t h} \sum_{\substack{\left.v_{j} \notin \operatorname{Path}^{\prime} \\
\& v_{j} \in \operatorname{Padjk}_{j}\right) \cup\left\{v_{j}\right\}}}\left(\left|K_{\Omega\left(v_{i}\right)}^{(i-1)}\right|-\left|K_{\Omega\left(v_{i}\right)}^{(j)}\right|\right)
\end{aligned}
$$

By some careful derivations, we can prove the above inequality.

It remains to evaluate the communication case.

Theorem 7. On solving a given sparse matrix, the communication time of multifrontal method is no more than that of column-, row-, and submatrix-Cholesky factorization.

Proof. This theorem can be proven if we can prove that for any path, say Path, from a leaf to the root on any elimination tree,

$$
\sum_{v_{j} \in \text { Path }} m s g_{C R S}\left(v_{j}\right) \geq \sum_{v_{i} \in \text { Path }} m s g_{M}\left(v_{i}\right) \text {. }
$$

By Lemmas 2, 3, the definitions of Madj, Padj, Mdeg, $P d e g$, and some properties of elimination tree, we have the following derivations:

$$
\begin{aligned}
& \sum_{v_{j} \in \text { Path }} m s g_{C R S}\left(v_{j}\right)=\sum_{v_{j} \in P_{a t h}} \sum_{k \text { Padj }\left(v_{j}\right)} 1 \\
& =\sum_{v_{j} \in \text { Path }} \sum_{\substack{v_{k} \in \text { Padj }\left(v_{j}\right) \\
\text { \& paren }\left(v_{k}\right) \in \text { Path }}} 1+\sum_{v_{j} \in \operatorname{Path}} \sum_{\begin{array}{c}
v_{k} \in \operatorname{Padj}\left(v_{j}\right) \\
\text { \& paren }\left(v_{k}\right) \in \operatorname{Path}
\end{array}} 1 \\
& \geq \sum_{v_{j} \in P a t h} \sum_{\substack{\left.v_{k} \in P \text { Padj( } v_{j}\right) \\
\text { \& paren }\left(v_{k}\right) \in P \text { Path }}} 1 \\
& =\sum_{p a r e n\left(v_{k}\right) \in P a t h v_{j} \in \operatorname{Madj}\left(v_{k}\right)} 1 \\
& =\sum_{\operatorname{paren}\left(v_{k}\right) \in \operatorname{Path} v_{j} \in \operatorname{Madj}\left(v_{k}\right)} 1 \\
& =\sum_{v_{i} \in P a t h v_{k} \in \operatorname{child}\left(v_{i}\right)} \sum_{v_{j} \in \operatorname{Madj}\left(v_{k}\right)} 1=\sum_{v_{t} \in \operatorname{Path}} m_{M} g_{M}\left(v_{i}\right)
\end{aligned}
$$

As a brief summary of this subsection, we conclude:

(1) Submatrix-Cholesky (multifrontal) consumes the least parallel computation time, then the columnCholesky and last the row-Cholesky.
(2) Multifrontal method suffers less parallel communication time than the other three methods, which suffer the same amount.

(3) To conclude, the multifrontal method is superior to the other three Cholesky factorization methods.

\subsection{An example problem}

In this subsection we illustrate the above evaluation with a simple problem whose Cholesky factor is shown in Figure 1. Figure 2 shows the $\left(m t p_{C}, m t p_{R}, m t p_{S M}\right)$ and $\left[m s g_{C R S}, m s g_{M}\right]$ for each node of the corresponding elimination tree. A simple accumulating yields $\operatorname{Comp}_{C}$ : $\operatorname{Comp}_{R}: \operatorname{Comp}_{S M}=48: 62: 30$ and $\operatorname{Comm}_{C R S}: \operatorname{Comp}_{M}=$ $19: 15$.

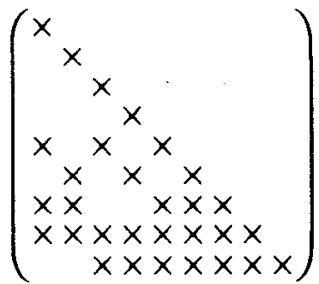

Figure 1. An example Cholesky factor.

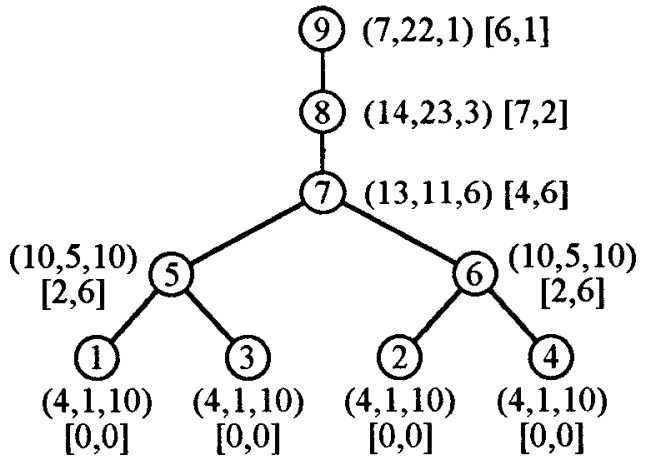

Figure 2. $\left(m t p_{C}, m t p_{R}, m t p_{S M}\right)$ and $\left[m s g_{C R S}, m s g_{M}\right]$.

This example reveals that even for a simple problem a great variation exists in the performance of different Cholesky factorization methods. Since most real world problems have large sizes and sophisticated structures, we can expect a tremendous variation and we conjecture the variation may become infinite to the extreme.

Moreover, no matter which form of Cholesky factorization algorithms is used, the total number of multiplicative operations as well as the message transfer is the same. Thus the above example reveals an interesting but important phenomenon: the submatrix-Cholesky (and multifrontal) leads to the best load balancing in view of coarse-grained task scheduling, then the columnCholesky, and last the row-Cholesky, which tells the reason of the theoretical results we obtained. 


\subsection{Experimental results}

We make an experiment on a set of test matrices from the well-known Harwell-Boeing sparse matrices collection; the choice is following [8]. All matrices have been ordered by the minimum degree ordering [5] to reduce the number of fill-ins. The experimental results are reported in Table 1. It can be seen that the results have assented the theoretical evaluation in Section 4.1 and it seems there is a limitation on the ratio between different methods. But what the variation would be remains unknown and needs more theoretical study.

\section{Conclusions}

In this paper, we have defined, formalized the criteria and evaluated the performance of column-, row- and submatrix-Cholesky, and the multifrontal method. In the theoretical study, we have observed that the well loadbalancing feature of multifrontal method makes it the supreme of the four methods.

However, the theoretical evaluation in this paper has some limitations. First, we only consider the coarsegrained task granularity (as exploited by elimination tree). In most practical implementations of parallel sparse Cholesky factorizations, they exploit the so called medium-grained granularity [11], and even fine-grained granularity [6]. Both cases are more complicated and deserved an advanced study. In addition, to reflect some subtle but influential overhead such as synchronization, indirect addressing and memory traffic, we also need a more sophisticated evaluation model.

\section{References}

[1] J. J. Dongarra, F. G. Gustavson and A. Karp, "Implementing linear algebra algorithms for dense matrices on a vector pipeline machine," SLAM Review 26, 1984, pp. 91112.

[2] I. S. Duff and J. K. Reid, "The multifrontal solution of indefinite sparse symmetric linear equations," $A C M$ Trans. Math. Software 9, 1983, pp. 302-325.

[3] I. S. Duff, "Parallel implementation of multifrontal schemes," Parallel Comput. 3, 1986, pp. 193-204.

[4] A. George, M. T. Heath and J. W. H. Liu, "Parallel Cholesky factorization on a shared-memory multiprocessor," Lin. Alg. Appl. 77, 1986, pp. 165-187.

[5] A. George and J. W. H. Liu, Computer Solution of Large Sparse Positive Definite Systems, Prentice Hall, Englewood Cliffs, NJ, 1981.

[6] J. R. Gilbert and R. Schreiber, "Highly parallel sparse Cholesky factorization," SLAM J. Sci. Stat. Comput. 13, 1992, pp. 1151-1172.

[7] M. T. Heath, E. Ng and B. W. Peyton, "Parallel algorithms for sparse linear systems," SIAM Review 33, 1991, pp. $420-460$

[8] J. G. Lewis, B. W. Peyton and A. Pothen, "A fast algorithm for reordering sparse matrices for parallel factorization," SIAM J. Sci. Stat. Comput. 10, 1989, pp. 11461173.

[9] W. Y. Lin and C. L. Chen, "Minimum completion time criterion for parallel sparse Cholesky factorization," in Proc. International Conference on Parallel Processing, Vol. III, St. Charles, IL, USA, 1993, pp. 107-114.

[10] W. Y. Lin and C. L. Chen, "Minimum communication time reordering for parallel sparse column-Cholesky factorization," in Proc. International Conference on Parallel And Distributed Systems, Taipei, Taiwan, 1993, pp. 147151.

[11] J. W. H. Liu, "Computational models and task scheduling for parallel sparse Cholesky factorization," Parallel Comput. 3, 1986, pp. 327-342.

[12] D. J. Rose, "A graph-theoretic study of the numerical solution of sparse positive definite systems of linear equations," in R. C. Read, ed., Graph Theory and Computing, Academic Press, New York, 1972, pp. 183-217.

[13] R. Schreiber, "A new implementation of sparse Gaussian elimination," ACM Trans. Math. Software 8, 1982, pp. 256-276.

Table 1. Statistics of the test problems.

\begin{tabular}{|c||r|r|r|r|r|r|r|}
\hline Key & Order & \#nonzeros & \multicolumn{1}{|c|}{ Comp $_{C}$} & \multicolumn{1}{|c|}{ Comp $_{R}$} & Comp $_{S M}$ & Comm $_{\text {CRS }}$ & Comm $_{M}$ \\
\hline \hline BCSPWR09 & 1723 & 2394 & 6089 & 8695 & 3616 & 1574 & 799 \\
BCSPWR10 & 5300 & 8271 & 50562 & 72586 & 32066 & 6606 & 2879 \\
BCSSTK08 & 1074 & 5943 & 784697 & 863997 & 620831 & 25373 & 16677 \\
BCSSTK13 & 2003 & 40940 & 22051166 & 26897928 & 15430855 & 216448 & 128960 \\
BCSSTM13 & 2003 & 9970 & 1850339 & 2069258 & 1283169 & 38433 & 24208 \\
BLCKHOLE & 2132 & 6370 & 762742 & 1022696 & 476237 & 27025 & 12630 \\
CAN 1072 & 1072 & 5686 & 151533 & 205136 & 103412 & 9762 & 4992 \\
DWT 2680 & 2680 & 11173 & 448620 & 584201 & 292997 & 29538 & 14719 \\
LSHP3466 & 3466 & 10215 & 1156859 & 1546570 & 720903 & 41703 & 19512 \\
GR 3030 & 900 & 4322 & 123050 & 172061 & 73056 & 8694 & 4039 \\
\hline
\end{tabular}

